\title{
Estrés en profesores de enseñanza secundaria: un análisis desde el optimismo
}

\author{
Estíbaliz Villardefrancos Pol, María José Santiago Mariño, Cristina \\ Castro Bolaño, Sofía Aché Reinoso y José Manuel Otero-López \\ Universidad de Santiago de Compostela (España)
}

\begin{abstract}
En los últimos años parece existir un notable consenso acerca de la importancia de las conductas y/o actitudes problemáticas de los alumnos, las situaciones conflictivas y la ausencia de apoyo social como factores que dificultan la convivencia escolar y contribuyen a incrementar el malestar laboral de los docentes. No obstante, son escasos los estudios empíricos realizados en torno a las relaciones entre dichas casuísticas y las variables de personalidad. El propósito de esta investigación es, a partir de una muestra de 1537 profesores de Enseñanza Secundaria Obligatoria, identificar cuáles son las fuentes de estrés que establecen diferencias significativas entre distintos niveles de optimismo (bajo, moderado y alto) autoinformado por los docentes; se analiza, también, si las facetas de estrés (conductas problemáticas de los alumnos, ausencia de apoyo social y conflicto) discriminan entre los profesores que informan de bajo vs. alto optimismo. Los resultados permiten concluir que los estresores considerados diferencian válidamente entre los niveles de optimismo. Se constata, asimismo, que las facetas conflicto y conductas problemáticas de los alumnos permiten discriminar en función del optimismo de los profesores.
\end{abstract}

Palabras clave: Estresores, optimismo, profesores de secundaria.

Stress in teachers in secondary education: an analysis from the optimism. There seems to be a remarkable consensus in recent years with regards to the importance of student disruptive behaviors and/or attitudes, conflictive situations, and the lack of social support as factors hindering school life and contributing to the increase of teacher occupational malaise. However, there are few empirical studies about the relationships between said causes and personality variables. The aim of this research based on a sample of 1537 secondary education teachers was to identify which sources of stress establish significant differences between levels of optimism (low, moderate and high) derived from teachers' self-reports; also analyzed was if the facets of stress (student disruptive behaviors, lack of social support and conflict) discriminate between teachers who report low vs. high optimism. The results suggest that the considered stressors validly differentiate between levels of optimism. Likewise, student disruptive behaviors and conflict facets allow for discrimination based on teacher optimism.

Key words: Stressors, optimism, secondary education teachers.

Correspondencia: José Manuel Otero-López. Departamento de Psicología Clínica y Psicobiología. Facultad de Psicología. Universidad de Santiago de Compostela. Campus Vida, Santiago de Compostela. C.P. 15782. A Coruña (España). E-mail: josemanuel.otero.lopez@ usc.es 
El malestar laboral de los docentes y educadores se ha erigido, a lo largo de las últimas décadas, como uno de los tópicos que parece haber suscitado especial atención e interés entre los miembros de la comunidad científica. En este sentido, diversas investigaciones han señalado a los profesores, en particular aquellos que desarrollan su labor en la enseñanza secundaria (ej., OCDE, 2009), como uno de los colectivos más vulnerables al desarrollo de estrés laboral y burnout.

En la búsqueda de los principales determinantes implicados en el estrés laboral de los docentes y que, a la postre, permitirían iluminar los posibles focos de actuación a nivel preventivo e interventivo, los esfuerzos parecen haberse dirigido fundamentalmente a la identificación y análisis de aquellos aspectos que dificultarían sobremanera la convivencia escolar. Así, desde distintas líneas de trabajo, se ha constatado que los comportamientos inadecuados de los alumnos tales como agresiones y vandalismo (ej., Kyriacou, 2003), las dificultades en el manejo de los conflictos surgidos entre los "actores" que intervienen en la escena educativa (ej., alumnos, padres, autoridades académicas) y el escaso apoyo social percibido por el docente (ej., Griffith, Steptoe y Cropley, 1999), constituyen algunos de los principales argumentos explicativos del malestar laboral en esta profesión. Más concretamente, en un reciente estudio desarrollado a partir de una amplia muestra de profesores de enseñanza secundaria (Otero-López, Santiago, Castro y Villardefrancos, 2010) se constató que las conductas problemáticas de los alumnos, la percepción de conflicto en los centros educativos y la ausencia de apoyo social, constituían las dimensiones en torno a las que se agrupaban algunos de los estresores laborales que adquirían mayor relevancia e impacto en los niveles de estrés experimentados por los docentes.

Si bien la literatura previa es sólida en cuanto a la fortaleza explicativa de las fuentes de estrés -es decir, los determinantes exógenos- en el malestar laboral y el burnout; no es menos cierto que, al amparo de la multicausalidad de estos fenómenos, también se ha desarrollado una prometedora y fecunda trayectoria de investigación orientada al estudio de las variables personales que pudiesen estar modulando el efecto negativo que los estresores ejercen en el individuo. En esta línea, y a pesar de la amplia plétora de determinantes analizados (ej., patrón de conducta Tipo A, locus de control), cabe reseñar que durante los últimos años han sido precisamente las variables incardinadas con la psicología positiva las que han generado algunos de los hallazgos más sugerentes. Sirvan de ejemplo, a este respecto, los trabajos que han documentado el papel de distintos constructos "positivos" tales como el optimismo (ej., Mäkikangas y Kinnunen, 2003; Otero-López y cols., 2008) y la personalidad resistente (ej., Moreno, Morett, Rodríguez y Morante, 2006; Otero-López, Castro, Santiago y Villardefrancos, 2010) como factores de protección frente al estrés y el burnout.

En concreto, el optimismo, definido por Scheier y Carver (1987) como la expectativa o creencia estable y generalizada en cuanto a la ocurrencia de resultados 
positivos en el futuro, emerge como una de las variables personales con mayor valor heurístico en el ámbito del malestar laboral. En efecto, la evidencia previa no sólo es consistente en cuanto a los efectos beneficiosos del optimismo a nivel físico y psicológico (ver, para una revisión, Rasmussen, Scheier, y Greenhouse, 2009); sino también en relación con su capacidad para reducir los niveles de estrés percibido. Así, por ejemplo, Paulik (2001) constató que el optimismo amortiguaba el impacto negativo de los estresores laborales en un grupo de profesores de universidad. En la misma línea, Mäkikangas y Kinnunen (2003) confirmaron, a partir de una amplia muestra de trabajadoras, que este determinante personal mitigaba el efecto de fuentes de estrés tales como la presión temporal, la inseguridad en el trabajo y el pobre clima organizacional. El optimismo también ha mostrado sólidos vínculos con otros indicadores de malestar laboral tales como la insatisfacción laboral y el burnout. A este respecto, en un reciente meta-análisis (Alarcón, Eschleman y Bowling, 2009) se constató que el optimismo covariaba negativamente con las dimensiones de cansancio emocional y despersonalización del burnout y positivamente con la faceta logro personal. Este patrón de hallazgos se ha obtenido también en algunos trabajos desarrollados con muestras de docentes de enseñanza primaria y secundaria (ej., Extremera, Durán y Rey, 2010). Asimismo, algunos estudios realizados con profesores de universidad han documentado la capacidad predictiva del optimismo en todas y cada una de las dimensiones del burnout (ej., Otero-López, Santiago y Castro, 2008). Finalmente, cabe reseñar que, a tenor de la fortaleza explicativa de esta variable y la solidez de sus vínculos con el estrés y el burnout, algunos autores (ej., Otero-López, Santiago y cols., 2010) han desarrollado tímidas propuestas explicativas en las que se postulaba al optimismo como posible variable moduladora del impacto de los estresores laborales en el síndrome de burnout.

En definitiva, esta investigación, con un decidido afán de consolidar el conocimiento acerca de los vínculos entre los distintos estresores que afectan a la convivencia escolar y el optimismo, se articula en torno a dos objetivos fundamentales: (a) esclarecer cuáles son las fuentes de estrés y las facetas (específicamente, conductas problemáticas de los alumnos, ausencia de apoyo social y conflicto) que diferencian, a niveles estadísticamente significativos, entre distintos niveles de optimismo autoinformado por los docentes de enseñanza secundaria; y, (b) determinar si las distintas facetas de estrés que dificultan la convivencia permiten discriminar entre los profesores que informan de bajo vs. alto optimismo.

\section{MÉTODO}

\section{Participantes}

La muestra está conformada por un total de 1537 profesores de Enseñanza Secundaria Obligatoria (ESO). Ésta es representativa de la Comunidad Autónoma de 
Galicia y se ha distribuido por tipologías de centros (IES y CPI), por hábitat (urbano, rural costero y rural interior) y por género (para más detalles, ver Otero-López y cols., 2012). En cuanto a las características de los participantes, destacamos las siguientes: 1006 son profesoras $(65.5 \%)$ y 531 profesores $(34.5 \%)$, el rango de edad oscila entre 26 y 65 años (edad media: 43.9 años), la antigüedad en la profesión se sitúa entre 3 y 32 años (media: 16.9 años), y el 39.7\% imparten docencia en primer ciclo de ESO, mientras que el $60.3 \%$ lo hace en segundo ciclo.

\section{Instrumentos}

Los profesores cumplimentaron el Inventario de Estresores Laborales para Profesores de Secundaria (IELPS; Otero-López y cols., 2012). Esta medida de autoinforme está integrada por 78 ítems que evalúan un amplio rango de situaciones relacionadas con distintos ámbitos de la labor docente y a los que debe responderse en función del grado de estrés que estas suelen suscitar en una escala de respuesta que oscila entre 0 (no produce tensión) y 4 (mucha tensión). La selección de los ítems para la presente investigación se realizó atendiendo a dos razones: por un lado, se incluyeron únicamente aquellos ítems que los docentes informaban que les generaban "muy alto" estrés y, por otro, se rescataron sólo aquellos enunciados que, a tenor de la literatura revisada (Travers y Cooper, 1997) y a partir del acuerdo interjueces, eran representativos de los dominios conceptuales a los que se pretendía dar cabida (conductas problemáticas de los alumnos, ausencia de apoyo social y conflicto); en definitiva, se trataba de incluir aquellas fuentes de estrés que dificultaban, en mayor medida, la convivencia escolar. En un segundo momento estos ítems se sometieron a un análisis factorial exploratorio (componentes principales, rotación varimax) obteniéndose una solución factorial coincidente con los conjuntos lógicos establecidos a priori (ver, para más detalles, Otero-López, Santiago y cols., 2010). Por último, cabe señalar que en este trabajo se analizarán no sólo los ítems correspondientes a cada factor, sino también los factores o dimensiones resultantes.

La versión revisada del Life Orientation Test (LOT-R; Scheier, Carver y Bridges, 1994) fue el instrumento seleccionado para medir el optimismo disposicional. El LOT-R consta de 10 ítems a los que se responde a partir de una escala de respuesta tipo Likert que oscila entre 0 (totalmente en desacuerdo) y 3 (totalmente de acuerdo). El coeficiente alpha de Cronbach fue .78 .

\section{RESULTADOS}

En este estudio, y en consonancia con los objetivos de partida, se ha realizado un análisis de varianza (ANOVA) para determinar qué fuentes de estrés y dimensiones establecen diferencias significativas en función del nivel de optimismo autoinformado 
(bajo, moderado y alto) por los docentes de enseñanza secundaria. Se ha aplicado, además, la prueba a posteriori de Scheffé para clarificar entre qué grupos -tomados dos a dos- se establecen dichas diferencias significativas.

Respecto a la dimensión conductas problemáticas de los alumnos, los resultados (ver Tabla 1) confirman que todos los estresores considerados permiten diferenciar a niveles estadísticamente significativos entre los grupos. Más específicamente, "las agresiones verbales por parte de los alumnos", "el que los alumnos intenten 'probarte' a cada instante" y "el vandalismo en las instalaciones del centro" son los estresores que presentan mayores valores de F (el rango oscila entre 55.45 y 49.14, $\mathrm{p}<.001)$. Los resultados de la prueba de Scheffé indican que todas y cada una de las fuentes de estrés pertenecientes a esta faceta establecen diferencias significativas y en la dirección esperada entre todas las posibles combinaciones de grupos tomadas dos a dos (comparaciones 1/2,1/3 y 2/3). Son, por tanto, aquellos profesores menos optimistas (grupo 1) -frente a los que obtienen puntuaciones moderadas y altas (grupos 2 y 3 , respectivamente)- los que presentan un mayor impacto percibido ante los estresores relacionados con las conductas problemáticas de los alumnos.

En cuanto a la faceta ausencia de apoyo social, nuestros hallazgos indican que cuatro de las cinco fuentes de estrés consideradas permiten diferenciar a niveles estadísticamente significativos entre los docentes con bajo, moderado y alto optimismo (la única excepción es el ítem "la falta de apoyo en algunas 'cuestiones' por parte de mi jefe de departamento"). Las diferencias responden al siguiente patrón: los profesores que presentan mayores niveles de optimismo son, cuando se les compara con los restantes grupos, los que informan de menores niveles de estrés ante las casuísticas referidas a ausencia de apoyo. En concreto, las mayores diferencias significativas inter-grupo corresponden al estresor "el no tener a quién recurrir cuando tengo un problema" ( $\mathrm{F}=$ 22.14, p<.001); además, este ítem es el único que, a tenor de los resultados de la prueba a posteriori de Scheffé, permite diferenciar válidamente entre todos los grupos. Los demás indicadores de ausencia de apoyo social establecen diferencias significativas entre los docentes con bajo y alto optimismo (comparación 1/3) y aquellos con moderado y alto optimismo (comparación 2/3).

Todos los estresores pertenecientes a la dimensión conflicto establecen diferencias estadísticamente significativas entre los grupos, constatándose mediante la prueba a posteriori de Scheffé que dichas diferencias se producen entre todas las posibles combinaciones de grupos tomados dos a dos. En un análisis más exhaustivo, cabe reseñar que las diferencias más acusadas, a tenor de los valores de $\mathrm{F}$, corresponden a las fuentes de estrés "el tener que reunirme con los padres de los alumnos conflictivos" $(\mathrm{F}=76.54, \mathrm{p}<.001)$, "la relación con los padres y madres de los alumnos" $(\mathrm{F}=52.24$, $\mathrm{p}<.001) \mathrm{y}$ "la falta de consenso entre el profesorado en asuntos disciplinarios" ( $\mathrm{F}=42.46$, $\mathrm{p}<.001)$. Así, los profesores con niveles bajos de optimismo informan, frente a los que 
presentan niveles moderados y altos, de un mayor nivel de estrés en relación con las casuísticas correspondientes a la faceta conflicto.

Tabla 1. Análisis de varianza en función del optimismo

\begin{tabular}{|c|c|c|c|c|c|c|}
\hline & & $\stackrel{\circ}{\overparen{\mathscr{F}}}$ & 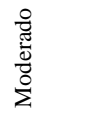 & $\stackrel{\ominus}{\stackrel{2}{4}}$ & 垊 & 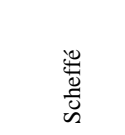 \\
\hline & & $N=391$ & $N=758$ & $N=388$ & & \\
\hline \multirow{6}{*}{ 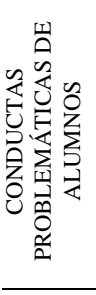 } & Las agresiones verbales por parte de los alumnos & 3.66 & 3.13 & 2.79 & $55.45 * * *$ & $1 / 2,1 / 3,2 / 3$ \\
\hline & El incremento de las agresiones entre los alumnos & 3.29 & 2.93 & 2.65 & $27.50 * * *$ & $1 / 2,1 / 3,2 / 3$ \\
\hline & El vandalismo en las instalaciones del centro & 3.40 & 2.80 & 2.49 & $49.14 * * *$ & $1 / 2,1 / 3,2 / 3$ \\
\hline & $\begin{array}{l}\text { La existencia de comportamientos y/o actitudes racistas } \\
\text { dentro del centro }\end{array}$ & 2.33 & 2.08 & 1.82 & $11.22 * * *$ & $1 / 2,1 / 3,2 / 3$ \\
\hline & $\begin{array}{l}\text { El que los alumnos adopten una actitud pasiva en las } \\
\text { clases }\end{array}$ & 3.18 & 2.79 & 2.47 & $31.95 * * *$ & $1 / 2,1 / 3,2 / 3$ \\
\hline & El que los alumnos intenten "probarte" a cada instante & 3.39 & 2.80 & 2.53 & $52.65 * * *$ & $1 / 2,1 / 3,2 / 3$ \\
\hline \multirow{5}{*}{ 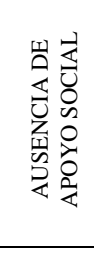 } & La falta de "apoyo social" por parte de mis colegas & 2.28 & 2.15 & 1.93 & $7.19 * * *$ & $1 / 3,2 / 3$ \\
\hline & $\begin{array}{l}\text { La falta de apoyo de algunas familias en asuntos } \\
\text { disciplinarios }\end{array}$ & 1.58 & 1.51 & 1.25 & $6.25 * * *$ & $1 / 3,2 / 3$ \\
\hline & El no tener a quién recurrir cuando tengo un problema & 2.59 & 2.36 & 1.95 & $22.14 * * *$ & $1 / 2,1 / 3,2 / 3$ \\
\hline & La falta de apoyo por parte de la dirección del centro & 2.11 & 2.09 & 1.71 & $10.40 * * *$ & $1 / 3,2 / 3$ \\
\hline & $\begin{array}{l}\text { La falta de apoyo en algunas "cuestiones" por parte de mi } \\
\text { jefe de departamento }\end{array}$ & 1.27 & 1.35 & 1.15 & n.s. & ---- \\
\hline \multirow{5}{*}{ 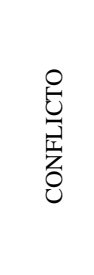 } & La relación con los padres y madres de los alumnos & 2.23 & 1.74 & 1.42 & $52.24 * * *$ & $1 / 2,1 / 3,2 / 3$ \\
\hline & $\begin{array}{l}\text { El tener que reunirme con los padres de los alumnos } \\
\text { conflictivos }\end{array}$ & 3.08 & 2.51 & 2.02 & $76.54 * * *$ & $1 / 2,1 / 3,2 / 3$ \\
\hline & $\begin{array}{l}\text { Los conflictos entre mi departamento y otros en el reparto } \\
\text { de recursos }\end{array}$ & 1.91 & 1.43 & 1.08 & $36.50 * * *$ & $1 / 2,1 / 3,2 / 3$ \\
\hline & La competitividad entre los profesores del centro & 1.76 & 1.49 & 1.15 & $19.57 * * *$ & $1 / 2,1 / 3,2 / 3$ \\
\hline & $\begin{array}{l}\text { La falta de consenso entre el profesorado en asuntos } \\
\text { disciplinarios }\end{array}$ & 3.22 & 2.80 & 2.49 & $42.46^{* * *}$ & $1 / 2,1 / 3,2 / 3$ \\
\hline
\end{tabular}

Por último, cuando se someten a examen las dimensiones conductas problemáticas de los alumnos, ausencia de apoyo social y conflicto, los resultados confirman las tendencias derivadas del análisis de las fuentes de estrés pertenecientes a cada una de ellas. Específicamente, se constata que la faceta conflicto es la que establece las mayores diferencias entre los grupos $(\mathrm{F}=76.19, \mathrm{p}<.001)$, seguida de las conductas problemáticas de los alumnos $(\mathrm{F}=55.77, \mathrm{p}<.001)$ y la ausencia de apoyo social $(\mathrm{F}=$ $13.15, \mathrm{p}<.001)$. Los resultados de la prueba a posteriori de Scheffé ponen de manifiesto que, mientras las dimensiones conductas problemáticas de los alumnos y conflicto permiten diferenciar entre los tres grupos considerados, la faceta ausencia de apoyo 
social da cuenta de las diferencias entre los docentes con bajos y altos niveles de optimismo y aquellos con moderado y alto optimismo. En cualquier caso y tal y como se avanzaba, el análisis de las dimensiones ratifica los hallazgos obtenidos a partir de la consideración de los distintos estresores, de tal manera que son, de nuevo, los profesores con menores niveles de optimismo autoinformado los que experimentan un mayor impacto o grado de estrés asociado a todas y cada una de las facetas.

A continuación, y con el objetivo de esclarecer qué dimensiones, cuando se consideran conjuntamente, permiten discriminar entre los grupos de docentes con bajo vs. alto optimismo establecidos a priori, se ha realizado un análisis discriminante. Tal y como se puede observar en la Tabla 2, el análisis selecciona en el primer paso la dimensión conflicto $(\mathrm{F}=138.992, \mathrm{p}<.001)$. Una vez extraída la faceta conflicto, el análisis selecciona en segundo lugar (paso 2) las conductas problemáticas de los alumnos $(\mathrm{F}=85.689, \mathrm{p}<.001)$, dimensión que completa la ecuación discriminante. Las facetas conflicto y conductas problemáticas de los alumnos permiten, entonces, clasificar correctamente a los profesores de la muestra en los grupos de bajo vs. alto optimismo; en concreto, se clasificaría correctamente al $73.1 \%$ de los docentes bajos en optimismo y al $67.3 \%$ de los que presentan elevadas puntuaciones en esta variable personal (media de casos clasificados correctamente $=70.2 \%$ ).

Tabla 2. Análisis discriminante para las distintas dimensiones de estresores en función del nivel de optimismo (bajo vs. alto)

\begin{tabular}{|c|c|c|c|c|c|c|}
\hline \multirow[b]{3}{*}{ Pasos } & \multirow{3}{*}{\multicolumn{2}{|c|}{ Estresores seleccionados }} & \multicolumn{4}{|c|}{ Lambda de Wilks } \\
\hline & & & \multirow[b]{2}{*}{ Estadístico } & & \multicolumn{2}{|c|}{$F$} \\
\hline & & & & & Estadístico & $\begin{array}{c}\text { Nivel de } \\
\text { significación }\end{array}$ \\
\hline 1 & Conflicto & & .848 & & 138.992 & .001 \\
\hline 2 & $\begin{array}{l}\text { Conductas prob } \\
\text { alumnos }\end{array}$ & & .819 & & 85.689 & .001 \\
\hline \multicolumn{7}{|c|}{ Autovalor =.221; Correlación canónica $=.426$} \\
\hline \multicolumn{7}{|c|}{ Número y porcentaje de casos clasificados correctamente } \\
\hline & \multicolumn{4}{|c|}{ Grupo de pertenencia pronosticado } & \multirow{2}{*}{\multicolumn{2}{|c|}{ Total }} \\
\hline & \multicolumn{2}{|c|}{ Grupo 1 Bajo optimismo } & \multicolumn{2}{|c|}{ Grupo 2 Alto optimismo } & & \\
\hline & $N$ & $\%$ & $N$ & $\%$ & $N$ & $\%$ \\
\hline $\begin{array}{c}\text { Grupo I Bajo } \\
\text { optimismo }\end{array}$ & 285 & $73.1 \%$ & 106 & $26.9 \%$ & 391 & 100 \\
\hline $\begin{array}{c}\text { Grupo 2 Alto } \\
\text { optimismo }\end{array}$ & 127 & $32.7 \%$ & 261 & $67.3 \%$ & 388 & 100 \\
\hline \multicolumn{7}{|c|}{ Media de casos clasificados correctamente $=70.2 \%$} \\
\hline
\end{tabular}

A tenor de estos hallazgos, cabría concluir que son precisamente aquellos profesores menos optimistas los que perciben como especialmente estresantes los conflictos derivados de las relaciones con los padres de los alumnos y/o la falta de acuerdo con otros docentes en relación a asuntos disciplinarios; asimismo, estos 
muestran una mayor sensibilidad o impacto ante las agresiones verbales por parte de los alumnos, el hecho de que estos intenten ponerles a prueba constantemente y/o el vandalismo en las instalaciones del centro.

\section{DISCUSIÓN}

Los resultados obtenidos en cuanto al primer objetivo de esta investigación confirman que, en general, las distintas fuentes de estrés que dificultan la convivencia escolar, así como las dimensiones en las que estas se agrupan (conductas problemáticas de los alumnos, conflicto y ausencia de apoyo social), diferencian a niveles estadísticamente significativos entre los tres grupos de docentes establecidos en base al optimismo autoinformado. Más concretamente, del análisis de varianza realizado se desprende que los docentes con elevado optimismo informan, frente a los que presentan bajo y moderado optimismo, de un menor impacto o nivel de tensión en relación con todos los estresores considerados.

Atendiendo a cada una de las facetas analizadas en este estudio, se constata que son las fuentes de estrés pertenecientes a la dimensión conflicto las que establecen las diferencias más acusadas en cuanto a la perspectiva del docente de que "las cosas le irán bien en el futuro" (Scheier y Carver, 1987). A este respecto, y aunque todas las fuentes de estrés a las que da cobertura esta faceta permiten diferenciar entre los distintos grupos de profesores, son los estresores vinculados a las relaciones con los padres de los alumnos (en especial, las reuniones con los padres de alumnos conflictivos) y el desacuerdo entre los propios docentes en relación con las cuestiones disciplinarias, los aspectos que permiten diferenciar en mayor medida entre los profesores que informan de alto, moderado y bajo optimismo; siendo, en todos los casos, los docentes menos optimistas los que experimentan un mayor nivel de estrés ante estas circunstancias. Estos hallazgos son consistentes con los obtenidos en algunas de las investigaciones más recientes en el ámbito del malestar laboral docente (ej., Otero-López, Castro, Villardefrancos y Santiago, 2009). Así, Bauer y cols. (2007) constataron que las interacciones negativas con los padres de los alumnos, en particular las quejas y acusaciones por parte de estos, constituían algunos de los más importantes estresores referidos por una amplia muestra de profesores de primaria y secundaria. De manera similar, y considerando un grupo de docentes de estos mismos niveles educativos, Gomes, Montenegro, Peixoto y Peixoto (2010) confirmaron que las inadecuadas políticas disciplinarias en la enseñanza incrementaban de manera notable los niveles de estrés entre el profesorado.

Los estresores incluidos bajo la dimensión conductas problemáticas de los alumnos también han puesto de manifiesto su capacidad para diferenciar entre los grupos de docentes establecidos en función del optimismo, siendo las agresiones verbales al 
profesor, las actitudes desafiantes y el vandalismo, las modalidades que establecen las mayores diferencias significativas. Resultan de interés, a este respecto, las aportaciones de Kyriacou (2003) al hacerse eco de la situación de "alerta constante" en la que parecen hallarse algunos profesores ante cualquier indicio de conductas disruptivas en el aula. Evidencia complementaria, aunque indirecta, a estos hallazgos se desprende del trabajo de Szalma (2009) que, a partir de la comparación entre optimistas y pesimistas en tareas de vigilancia, concluyó que estos últimos mostraban un estado de "activación" permanente que incrementaba sustancialmente los niveles de estrés experimentados durante la tarea. Pues bien, en un afán de discutir nuestros hallazgos, cabría pensar en la posibilidad de que sea precisamente el "optimismo disposicional" (Avia y Vázquez, 1998) el que permita a los profesores reducir los niveles de "alerta" y desarrollar cierta expectativa de control ante los diversos avatares derivados de la convivencia escolar, lo cual podría minimizar, en última instancia, el impacto negativo de los mismos.

En cuanto a la dimensión ausencia de apoyo social cabe señalar que, tal y como se avanzaba, la práctica totalidad de los estresores considerados bajo esta rúbrica permiten establecer diferencias entre los grupos de docentes con distintos niveles de optimismo, siendo los profesores pertenecientes al grupo de bajo optimismo los que informan de una mayor sensibilidad en relación con las casuísticas vinculadas a esta faceta. Estos resultados coinciden con los derivados de algunas investigaciones previas en el área del malestar laboral docente y en las que se ha constatado que los bajos niveles de apoyo social percibido por los profesores se vinculan con el estrés laboral y el burnout (ej., Griffith y cols., 1999). Atendiendo a la "otra cara de la moneda" o los efectos beneficiosos del apoyo, Pomaki, DeLongis, Frey, Short y Woehrle (2010) confirmaron que el apoyo social proporcionado por los compañeros incrementaba los niveles de satisfacción laboral de los docentes y reducía la intención de abandono de la profesión.

Por lo que respecta al segundo objetivo de este trabajo (dilucidar si las dimensiones de estrés consideradas permiten discriminar entre los profesores con bajo vs. alto optimismo), el conflicto emerge como la faceta con mayor protagonismo. La dimensión conductas problemáticas de los alumnos, como cabría esperar en función de los resultados previos, se consolida, después del conflicto, como otro de los argumentos empíricos para discriminar en función del optimismo. Parece razonable pensar entonces que, cuando enfrentamos a los docentes optimistas con los pesimistas, existan acusadas diferencias en el modo de encajar los conflictos y las conductas-problema de los alumnos; de modo que los juicios, el grado de tensión y, posiblemente, la respuesta sean mucho más "equilibrados" en aquellos que tienen la expectativa generalizada de que "todo va razonablemente bien".

Cabría concluir, a tenor de los objetivos de la presente investigación, que: 1) la práctica totalidad de los estresores que dificultan la convivencia escolar, así como las 
facetas a las que estos pertenecen -conductas problemáticas de los alumnos, ausencia de apoyo social y conflicto-, establecen diferencias significativas entre los distintos niveles de optimismo autoinformado por los docentes que conforman la muestra, y 2) las dimensiones conflicto y conductas problemáticas de los alumnos permiten discriminar entre los profesores con bajo vs. alto optimismo. En definitiva, cabría considerar que las "fortalezas" del docente optimista pudiesen radicar en que muy probablemente éste se muestre, frente al pesimista, más dispuesto a superar cualquier situación adversa (ej., conflictos, conductas problemáticas), perciba en menor medida la ausencia de apoyo social y se implique más activamente en la consolidación de redes de apoyo eficaces.

Para finalizar, y a la espera de que estos hallazgos sean ratificados en futuras investigaciones, sólo nos queda apuntar algunas de las potenciales debilidades del presente trabajo. Entre ellas, cabría destacar la autoselección de la muestra y el carácter eminentemente exploratorio del estudio, imposibilitándose cualquier intento de determinar relaciones causales. En este sentido, y en un afán de apuntar algunas directrices que probablemente contribuirían al avance del conocimiento en el campo, cabría señalar la oportunidad de integrar las fuentes de estrés consideradas y el optimismo en modelos explicativos del malestar laboral docente. Es decir, una vez constatada la existencia de vínculos entre los estresores y el optimismo, parece oportuno avanzar un paso más de cara a la comprobación empírica de modelos que contribuyan a clarificar el orden de influencias entre estas variables.

\section{REFERENCIAS}

Alarcón, G., Eschleman, K.J. y Bowling, N.A. (2009). Relationships between personality variables and burnout: A meta-analysis. Work \& Stress, 23, 244-263.

Avia, M.D. y Vázquez, C. (1998). Optimismo inteligente. Madrid: Alianza.

Bauer, J., Unterbrink, T., Hack, A., Pfeifer, R., Buhl-Griebhaber, V., Müller, U., Wesche, H., Frommhold, M., Seibt, R., Scheuch, K. y Wirsching, M. (2007). Working conditions, adverse events and mental health problems in a sample of 949 German teachers. International Archives of Occupational and Environmental Health, 80, 442-449.

Extremera, N., Durán, A. y Rey, L. (2010). Recursos personales, síndrome de estar quemado por el trabajo y sintomatología asociada al estrés en docentes de enseñanza primaria y secundaria. Ansiedad y Estrés, 16, 47-60.

Gomes, A.R., Montenegro, N., Peixoto, A.M. y Peixoto, A.R. (2010). Stress ocupacional no ensino: Un estudo com professores dos $3^{\circ}$ ciclo e ensino secundario. Psicologia \& Sociedade, 22, 587-597.

Griffith, J., Steptoe, A. y Cropley, M. (1999). An investigation of coping strategies associated with job stress in teachers. British Journal of Educational Psychology, 69, 517-531.

Kyriacou, C. (2003). Antiestrés para profesores. Barcelona: Octaedro (trabajo original publicado en 2001).

Mäkikangas, A. y Kinnunen, U. (2003). Psychosocial work stressors and well-being: Self-esteem and optimism as moderators in a one-year longitudinal sample. Personality and Individual Differences, 35, 537-557. 
Moreno, B., Morett, N.I., Rodríguez, A. y Morante, M.E. (2006). La personalidad resistente como variable moduladora del síndrome de burnout en una muestra de bomberos. Psicothema, $18,413-418$.

OCDE (2009). Informe TALIS. La creación de entornos eficaces de enseñanza y aprendizaje. Sintesis de los primeros resultados. OCDE: Santillana.

Otero-López, J.M., Castro, C., Santiago, M.J. y Villardefrancos, E. (2010). Exploring stress, burnout, and job dissatisfaction in secondary school teachers. International Journal of Psychology and Psychological Therapy, 10, 107-123.

Otero-López, J.M., Castro, C., Villardefrancos, E. y Santiago, M.J. (2009). Job dissatisfaction and burnout in secondary school teachers: student's disruptive behaviour and conflict management examined. European Journal of Education and Psychology, 2, 99-111.

Otero-López, J.M., Santiago, M.J. y Castro, C. (2008). An integrating approach to the study of burnout in university professors. Psicothema, 20, 766-772.

Otero-López, J.M., Santiago, M.J., Castro, C., Pardiñas, M.C., Mirón, L. y Ponte, D. (2012). Estrés laboral y burnout en profesores de Enseñanza Secundaria. Madrid: Díaz de Santos.

Otero-López, J.M., Santiago, M.J., Castro, C. y Villardefrancos, E. (2010). Stressors rendering school coexistence difficult, personal variables and burnout: towards an explanatory model. European Journal of Education and Psychology, 3, 299-316.

Otero-López, J.M., Santiago, M.J., Godás, A., Castro, C., Villardefrancos, E y Ponte, D. (2008). An integrative approach to burnout in secondary school teachers: Examining the role of student disruptive behaviour and disciplinary issues. International Journal of Psychology and Psychological Therapy, 8, 259-270.

Paulik, K. (2001). Hardiness, optimism, self-confidence and occupational stress among university teachers. Studia Psychologica, 43, 91-100.

Pomaki, G., Delongis, A., Frey, D., Short, K. y Woehrle, T. (2010). When the going gets though: Direct, buffering and indirect effects of social support on turnover intention. Teaching and Teacher Education, 26, 1340-1346.

Rasmussen, H.N., Scheier, M.F. y Greenhouse, J.B. (2009). Optimism and physical health: A meta-analytic review. Annals of Behavioral Medicine, 37, 239-256.

Scheier, M.F. y Carver, C.S. (1987). Dispositional optimism and physical well-being: The influence of generalized expectancies on health. Journal of Personality, 55, 169-210.

Scheier, M.F., Carver, C.S., y Bridges, M.W. (1994). Distinguishing optimism from neuroticism: A reevaluation of the Life Orientation Test. Journal of Personality and Social Psychology, 67, 1063-1078.

Szalma, J.L. (2009). Individual differences in performance, workload, and stress in sustained attention: Optimism and pessimism. Personality and Individual Differences, 47, 444451.

Travers, C.J. y Cooper, C.L. (1997). El estrés de los profesores. La presión en la actividad docente. Barcelona: Paidós (trabajo original publicado en 1996).

Recibido: 20 de noviembre de 2012

Recepción Modificaciones: 1 de diciembre de 2012

Aceptado: 2 de diciembre de 2012 